\title{
A PROGRAM TO DETERMINE AN ACCURATE TRIGONOMETRIC PARALLAX FOR THE HYADES CLUSTER
}

\author{
W. F. VAN ALTENA \\ Yerkes Observatory, University of Chicago, Williams Bay, Wisc., U.S.A.
}

\begin{abstract}
A list is presented of very probable Hyades cluster members that are suitable for an accurate determination of the cluster's trigonometric parallax.
\end{abstract}

In 1968 I prepared a list of very probable Hyades cluster members which were suitable for parallax determination in an effort to improve the distance to the Hyades cluster and the population I distance scale. This list (Table 1 ) contains 20 very probable Hyades members lying within $3^{\circ}$ of the cluster center. The criteria for membership are: (1) photoelectric photometry is available which indicates either main sequence or white dwarf membership; and (2) proper motions are available from at least three sources yielding errors of $\leqslant \pm 3^{\circ}$ (m.e.) and $\pm 0.006 \mathrm{yr}^{-1}$ (m.e.), These conditions are relaxed

TABLE I

Very probable Hyades members suitable for $\pi$ determination

\begin{tabular}{|c|c|c|c|c|c|c|c|c|c|c|c|}
\hline \multicolumn{12}{|c|}{ Main sequence } \\
\hline No. & $N$ & $\mu$ & $\sigma \mu$ & $\Delta \mu$ & $\theta$ & $\sigma \theta$ & $\Delta \theta$ & $V$ & $B-V$ & $U-B$ & $\begin{array}{l}\text { Remarks } \\
\text { Priority }\end{array}$ \\
\hline 276 & 5 & $0 ! 136$ & \pm 5 & +20 & $100^{\circ}$ & $\pm 2^{\circ}$ & -1 & 10.49 & 1.24 & 1.18 & 7 \\
\hline 294 & 4 & 0.126 & 6 & +12 & 97 & 3 & -1 & 10.90 & 1.30 & 1.30 & 8 \\
\hline 310 & 4 & 0.119 & 5 & +3 & 104 & 3 & -1 & 9.99 & 1.06 & 0.95 & 5 \\
\hline 363 & 3 & 0.112 & 5 & -3 & 106 & 3 & +1 & 9.01 & 0.94 & 0.66 double? & 14 \\
\hline 459 & 4 & 0.114 & 6 & +3 & 97 & 3 & -2 & 9.52 & 0.93 & 0.70 & 4 \\
\hline 472 & 4 & 0.118 & 6 & +8 & 101 & 3 & +3 & 9.03 & 0.84 & 0.51 & 2 \\
\hline 475 & 4 & 0.105 & 6 & -7 & 103 & 3 & -1 & 11.13 & 1.37 & 1.24 & 10 \\
\hline 500 & 4 & 0.101 & 5 & -10 & 99 & 3 & -4 & 10.71 & 1.36 & 1.18 double? & 15 \\
\hline 502 & 5 & 0.109 & 5 & -2 & 102 & 2 & -2 & 11.96 & 1.44 & 1.24 & 11 \\
\hline 529 & 5 & 0.111 & 6 & +1 & 102 & 3 & +1 & 12.44 & 1.47 & $1.02(1)$ & 13 \\
\hline 548 & 4 & 0.104 & 5 & -7 & 101 & 3 & -3 & 10.32 & 1.17 & 1.08 & 6 \\
\hline 560 & 4 & 0.115 & 5 & +4 & 104 & 3 & 0 & 9.10 & 0.85 & 0.58 & 3 \\
\hline 587 & 4 & 0.096 & 3 & -14 & 101 & 2 & -1 & 8.93 & 0.84 & 0.49 & 1 \\
\hline 638 & 5 & 0.106 & 6 & -2 & 97 & 3 & -5 & 12.17 & 1.47 & $1.00(1)$ & 12 \\
\hline 645 & 5 & 0.116 & 6 & +8 & 102 & 3 & 0 & 11.04 & 1.31 & 1.23 & 9 \\
\hline 747 & 4 & 0.092 & 4 & -14 & 99 & 2 & -4 & 9.69 & 0.91 & 0.68 & 16 \\
\hline \multicolumn{7}{|c|}{$0.000 \pm 1$} & \multicolumn{5}{|c|}{$-1^{\circ} \pm 1^{\circ}-0.04 \pm .06$} \\
\hline \multicolumn{12}{|c|}{ White dwarfs } \\
\hline 292 & 4 & 0.124 & 7 & +8 & 102 & 3 & 0 & 14.29 & -0.02 & -0.84 & 19 \\
\hline 490 & 4 & 0.101 & 8 & -15 & 103 & 4 & -2 & 14.02 & -0.09 & -0.97 & 17 \\
\hline 673 & 5 & 0.118 & 5 & +9 & 110 & 2 & +3 & 13.95 & +0.33 & -0.69 double & 20 \\
\hline 722 & 3 & 0.096 & 6 & -9 & 96 & 2 & -1 & 14.18 & -0.03 & -0.89 & 18 \\
\hline \multicolumn{7}{|c|}{$-0{ }^{\prime \prime 002} \pm 3$} & \multicolumn{3}{|c|}{$0^{\circ} \pm 2^{\circ}$} & & \\
\hline
\end{tabular}


in the case of the white dwarfs), and the position angle of the motion must lie within two mean errors of the convergent point.

Only the classical DA white dwarfs are included. As indicated in the table the average motion of these stars with respect to the Hyades motion is not statistically significant. The average difference of the 16 main sequence stars from the average Hyades main sequence is not statistically significant. The priorities are ordered by apparent magnitude, except for those stars either known to be double or suspected of being double, and number 747 which I feel has the weakest case for being a member. Cross identifications and finding charts may be found in Tables IIIA and IIIC and in Plate I of Astron. J. 74, 2, 1969.

At the present time seven Observatories (Allegheny, Cape, Herstmonceux, Lick, McCormick, Van Vleck, and Yerkes) are co-operating in this joint program which should yield an average parallax for the Hyades with an error of $\sim \pm 0$ ".001 (m.e.) for each observatory. Within the next few years it should be possible to determine an accurate parallax for the Hyades cluster from these data that will have approximately the same accuracy as that found from the convergent point method.

In addition to the primary aim of this project, it will also be possible to evaluate the systematic differences between observatories with modern data for the Hyades region of the sky.

\section{DISCUSSION}

Worley: Eggen commented that the double star orbits from which the masses of the Hyades binaries are determined result from observations made over a long period of time by observers of varying skill. How much this inhomogeneity affects the resultant masses is the question.

Together with a colleague G. G. Douglas, I have been examining the problem of the mass discrepancy of the Hyades binaries vs the 'normal' (Sun-Sirius) ML relation. Unless we are willing to accept a very peculiar systematic error, which affects the observations made in the region of the Hyades, but which does not appear in the rest of the sky, we cannot attribute the ML discrepancy to systematic effects in the observations.

The above investigation also pointed out that a more general study of systematic errors was needed, since the last such investigation was made many years ago. Such a study is now in progress.

Wesselink: The Leiden Observatory (Miss Kluyvers) is conducting a new study of proper motions convergent point, distance modulus of the Hyades. This study may give rise to an interesting new answer to the modulus problem.

Van Altena: A redetermination of the Hyades convergent point is being made from absolute proper motions with respect to faint galaxies by Robert Hanson at Lick Observatory.

Thomas: I have a question concerning the chemical composition of the Hyades derived with Watson opacities. Did I understand you correctly that these values stem from fitting the theoretical slopes of the mass-radius and mass-luminosity relation to the observations. Since chemical composition does not enter the homology relations, is this an effect of the non-homology of computed models?

Demarque: No. The theoretical slopes of the mass-radius and mass-luminosity relations are practically independent of chemical composition in the relevant mass range. The fitting was done by requiring consistency between the distance modulus used in deriving the masses of Hyades binaries and that obtained from the position of the main sequence.

Crawford: We find in our work that the Hyades is more discrepant than any other cluster. Any change in the distance modulus will not solve the problem. I will describe the discrepancy a bit tomorrow.

Pecker: Within clusters (Hyades, Praesepe...) is not the reddening correction to be questioned because of inter-cluster additional reddening? (a sort of circumstellar reddening?)

Eggen: There is no evidence for appreciable reddening either in the Hyades or in Praesepe. 